\title{
HOMOLOGICAL PROPERTIES OF COHERENT SEMILOCAL RINGS
}

\author{
CHENG FUCHANG, ZHAO YICAI, AND TANG GAOHUA
}

(Communicated by Louis J. Ratliff, Jr.)

\begin{abstract}
W. V. Vasconcelos and M. Auslander have studied the homological properties of coherent (noetherian) rings. In [3] some theorems for regular local rings were generalized to noetherian semilocal rings. The main aim of this paper is to discuss coherent semilocal rings.
\end{abstract}

\section{HoMologicAl DIMENSIONS OF COHERENT SEMILOCAL RINGS}

Let $R$ be a commutative ring with identity element, and let $J$ be the Jacobson radical of $R$. The concepts and the notations that are used in this paper are consistent with those in [14].

Definition. Let $A$ be an $R$-module. A normal $A$-sequence is an ordered sequence $u_{1}, u_{2}, \ldots, u_{n}$ in $J$ such that $u_{1}$ is not a zero divisor in $A$ and, for $i>1$, each $u_{i}$ is not a zero divisor in $A /\left(u_{1}, \ldots, u_{i-1}\right) A$. We write $\operatorname{cod}_{R}(A)=n$ if there exists a normal $A$-sequence with $n$ terms but no normal sequence with more than $n$ terms.

Theorem 1.1. Let $R$ be a coherent semilocal ring such that $J$ is finitely generated, and let $A$ be a finitely presented $R$-module. Then

(i) Tor-dim $R=p d_{R}(R / J)$ :

(ii) If $u_{1}, u_{2}, \ldots, u_{n}$ is a normal A-sequence, then

$$
p d_{R}\left(A / u\left(u_{1}, \ldots, u_{n}\right) A\right)=p d_{R}(A)+n .
$$

Proof. (i) Because $R / J$ is a finitely presented $R$-module and $R$ a coherent ring, $p d_{R}(R / J)=$ flat- $\operatorname{dim}_{R}(R / J) \leq$ Tor- $\operatorname{dim} R$ by [2, Proposition 2]. Assume that $p d_{R}(R / J)=n<\infty$. If $A$ is a finitely presented $R$-module, then $\operatorname{Tor}_{n+1}^{R}(A, R / J)=0$. Hence $\operatorname{Tor}_{n+1}^{R_{M}}\left(A_{M}, R_{M} / J_{M}\right)=0$, where $M$ is any maximal ideal of $R$. Since $R$ is a semilocal ring, $J_{M}=M_{M}$. Thus

$$
\operatorname{Tor}_{n+1}^{R_{M}}\left(A_{M}, R_{M} / M_{M}\right)=0
$$

Received by the editors July 27, 1989 and, in revised form, December 13, 1989.

1980 Mathematics Subject Classification (1985 Revision). Primary 13DXX. 
and by [14, Lemma 5.11], $p d_{R_{M}}\left(A_{M}\right) \leq n$. From [6, (3.E)] we have

$$
\text { flat- } \begin{aligned}
\operatorname{dim}_{R}(A) & =\sup \left\{\text { flat- } \operatorname{dim}_{R_{M}}\left(A_{M}\right) \mid M \text { is a maximal ideal of } R\right\} \\
& =\sup \left\{p d_{R_{M}}\left(A_{M}\right) \mid M \text { is a maximal ideal of } R\right\},
\end{aligned}
$$

which shows that flat- $\operatorname{dim}_{R}(A) \leq n$. On the other hand,

Tor- $\operatorname{dim} R=\sup \left\{\right.$ flat- $\operatorname{dim}_{R}(A) \mid A$ is a finitely presented $R$-module $\}$, hence

$$
\text { Tor- } \operatorname{dim} R=p d_{R}(R / J) \text {. }
$$

(ii) The statement can be shown in a proof similar to that of $[10$, Theorem 20, p. 196].

The Auslander-Buchsbaum Theorem affirms that if $A$ is a finitely generated module over a regular local ring $R$, then $\operatorname{pd}_{R}(A)+\operatorname{cod}_{R}(A)=\operatorname{gl}$.dim $R$. We give a similar result over a coherent local ring as follows.

Theorem 1.2. Let $R$ be a coherent local ring such that Tor- $\operatorname{dim} R<\infty$, and let $J$ be finitely generated. If $A$ is a finitely presented noetherian $R$-module, then $p d_{R}(A)+\operatorname{cod}_{R}(A)=$ Tor- $\operatorname{dim} R$.

Before proving Theorem 1.2, we first give

Lemma. Let $R$ be a coherent local ring such that Tor- $\operatorname{dim} R<\infty$, and let $J$ be finitely generated. Suppose that $A$ is a finitely presented noetherian $R$-module. Then

(i) If the only submodule $B$ of $A$ such that $J B=0$ is the zero submodule, then $J$ contains an element which is not a zero divisor in $A$.

(ii) If there exists a submodule $B \neq 0$ of $A$ such that $J B=0$, then $p d_{R}(A)=$ Tor- $\operatorname{dim} R$.

Proof. (i) See the proof of [9, Theorem 17, p. 186].

(ii) We may assume $B=(x)$. The homomorphism $R \rightarrow B$, in which $r \in R$ is mapped into $r x$, has kernel $J$, hence $B \cong R / J=F$, and then we have an exact sequence $0 \rightarrow F \rightarrow A \rightarrow A / B \rightarrow 0$. Assume Tor- $\operatorname{dim} R=p$, then $\operatorname{Tor}_{P+1}^{R}$ vanishes identically, and therefore $0 \rightarrow \operatorname{Tor}_{P}^{R}(F, F) \rightarrow \operatorname{Tor}_{P}^{R}(A, F)$ is exact. But $\operatorname{Tor}_{P}^{R}(F, F) \neq 0$, so $\operatorname{Tor}_{P}^{R}(A, F) \neq 0$, which shows that flat- $\operatorname{dim}_{R}(A) \geq p$. Since $A$ is finitely presented, flat- $\operatorname{dim}_{R}(A)=p d_{R}(A)$. Hence $p d_{R}(A)=$ Tor- $\operatorname{dim} R$.

Proof of Theorem 1.2. It is clear by Theorem 1.1 that $\operatorname{cod}_{R}(A) \leq$ Tor- $\operatorname{dim} R<$ $\infty$. Assume $\operatorname{cod}_{R}(A)=s$. Then there exists a normal $A$-sequence $u_{1}, \ldots, u_{s}$. By Theorem 1.1, we have

$$
\operatorname{cod}_{R}(A)+p d_{R}(A)=s+p d_{R}(A)=p d_{R}\left(A /\left(u_{1}, \ldots, u_{s}\right) A\right) .
$$

Now, we need only to show that $p d_{R}\left(A /\left(u_{1}, \ldots, u_{s}\right) A\right)=$ Tor- $\operatorname{dim} R$ and, by the Lemma, this will follow if we can show that $J$ annihilates some nonzero 
submodule of $A /\left(u_{1}, \ldots, u_{s}\right) A$. Assume the contrary. Since $A$ is a finitely presented noetherian $R$-module, so is $A /\left(u_{1}, \ldots, u_{s}\right) A$. By the Lemma there exists $u_{s+1} \in J$ such that $u_{s+1}$ is not a zero divisor in $A /\left(u_{1}, \ldots, u_{s}\right) A$. But this means that $u_{1}, \ldots, u_{s}, u_{s+1}$ is a normal $A$-sequence, and therefore $\operatorname{cod}_{R}(A) \geq s+1$, which contradicts the above hypothesis. Hence $\operatorname{cod}_{R}(A)+$ $p d_{R}(A)=$ Tor- $\operatorname{dim} R$.

\section{THE STRUCTURE OF COHERENT SEMILOCAL RINGS}

[14, Theorem 2.2] says that a local ring of global dimension 2 is either a regular local ring, a valuation domain, or an umbrella ring. Here we discuss coherent semilocal rings, and similar results will be obtained.

Theorem 2. Let $R$ be an indecomposable semilocal ring. If $R$ has the property that $\operatorname{gld} \operatorname{dim} R_{M} \leq r$ for each maximal ideal $M$, then $\operatorname{gl} \cdot \operatorname{dim} R \leq r^{(*)}$. Furthermore, if gl.dim $R=2$, then

(i) $R$ is a coherent GCD domain.

(ii) Every ideal of $R$ is countably generated.

(iii) $R$ must be one of the following: $a(1,2,3)$-ring, $a(2,2,0)$-ring, or a $(2,2,3)$-ring.

Remark. We say a ring $R$ is an $(a, b, c)$-ring provided that Tor- $\operatorname{dim} R=a$, gl.dim $R=b$, and f.p. $\operatorname{dim} R=c$ [8]. Before proving Theorem 2.1, we first give the following:

Proposition 2.1. Let $R$ be an indecomposable coherent semilocal ring. Then $R$ is a GCD domain if and only if $p d_{R}(a, b) \leq 1, \forall a, b \in R$.

Proof. Assume $R$ is a $G C D$ domain. $\forall a, b \in R$, if $(a, b)$ is a principal ideal, then $(a, b) \cong R$, and then $p d_{R}(A, b)=0$. From now on, we assume $(a, b)$ is not principal. Consider the exact sequence

$$
0 \rightarrow K \rightarrow R^{(2)} \stackrel{f}{\rightarrow}(a, b) \rightarrow 0,
$$

where $f(1,0)=a, f(0,1)=b$, and $K=\operatorname{Ker} f$. Obviously $K \cong(a) \cap(b)$. Let $[a, b]=d$. Then there exists $a^{\prime}, b^{\prime}$ in $R$ such that $a=d a^{\prime}, b=d b^{\prime}$, and $\left[a^{\prime}, b^{\prime}\right]=1$. Write $c=d a^{\prime} b^{\prime}$. Then $(c) \subseteq(a) \cap(b) . \forall x \in(a) \cap(b)$, there exist $r, s$ in $R$ such that $x=r a=s b$, and then $r a^{\prime} d=s b^{\prime} d$. Since $R$ is a domain, $r a^{\prime}=s b^{\prime}$. But $\left[a^{\prime}, b^{\prime}\right]=1$; thus, $b^{\prime} \mid r$. That is, there exists $r^{\prime}$ in $R$ such that $r=r^{\prime} b^{\prime}$. It follows that $x=r a=r a^{\prime} d=r^{\prime} b^{\prime} a^{\prime} d=r^{\prime} c$; that is, $x \in(c)$, and then $(a) \cap(b)=(c)$. Hence $K \cong(c)$ and so $K$ is a projective $R$-module, which shows that $p d_{R}(a, b) \leq 1$.

For the converse, $\forall 0 \neq a \in R$, from the exact sequence $0 \rightarrow \operatorname{Ann}(a) \rightarrow$ $R \rightarrow(a) \rightarrow 0$ and $p d_{R}(a) \leq 1$, we know that $\operatorname{Ann}(a)$ is finitely generated projective ideal, and then $\operatorname{Ann}(a)$ is free by [3, Corollary 1.2]. But $a \neq 0$, thus $\operatorname{Ann}(a)=0$, which means that $R$ is a domain. Furthermore, $\forall a, b \in R$, assume $(a, b)$ is not a principal ideal. Since $p d_{R}(a, b) \leq 1$ and $R$ is a 
coherent ring, $K$ is projective in the exact sequence (1), and then free. But $R$ is a domain and $K \cong(a) \cap(b)$; thus, $(a) \cap(b)$ is principal, which shows that $K$ is a free module with rank 1 . Let $K$ be generated by $\left(a_{1}, b_{1}\right)$. Since $f(-b, a)=-b a+a b=0,(-b, a) \in K$; that is, there exists $d$ in $R$ such that $(-b, a)=d\left(a_{1}, b_{1}\right)$. Hence $a=d a_{1}, b=-d b_{1}$. It is easy to verify that $d=[a, b]$. Consequently, $R$ is a $G C D$ domain.

Proposition 2.2. Let $R$ be a semilocal ring and $A$ an $R$-module. Then

(i) $A$ is finitely (countably) generated if and only if $A_{M}$ is a finitely (countably) generated $R$-module for each maximal ideal $M$ of $R$.

(ii) $R$ is a noetherian (coherent) ring if and only if $R_{M}$ is noetherian for each maximal ideal $M$ of $R$.

Proof. (i) We need only prove the sufficiency. Assume all maximal ideals of $R$ are $M_{1}, \ldots, M_{t}$ and $A_{M_{i}}$ be finitely generated $R_{M_{i}}$-module for each $M_{i}$. Let $X_{i}=\left\{x_{i t} / 1 \mid i_{t} \in T_{i}\right\}$ be a finitely generated set of $A_{M_{i}}$. Then $X=\bigcup_{i=1}^{t} X_{i}$ and $T=\bigcup_{i=1}^{t} T_{i}$ are finite sets, where $\bigcup$ means disjoint union. Define $f: R^{(T)} \rightarrow A$ by $f\left(e_{i_{t}}\right)=x_{i_{t}}$, where $e_{i_{t}}$ is that element having 1 in the $i_{t}$ th coordinate and 0 elsewhere. Obviously, $f$ is a $R$-homomorphism, and $f_{M_{i_{i}}}: R_{M_{i}}^{(T)} \rightarrow A_{M_{i}}$ is surjective. Consequently, $f$ is surjective, showing that $A$ is finitely generated.

Similarly for the countably generated case.

(ii) follows immediately from (i).

Proof of Theorem 2.1. If a semilocal ring $R$ has the property gl.dim $R_{M} \leq r$ for each maximal ideal $M$ then gl. $\operatorname{dim} R \leq r$. This follows from the descent of projectivity for faithfully flat morphisms (in the case $R \rightarrow R_{M_{1}} \times \cdots \times R_{M_{n}}$ ) of Gruson and Raynaud [11, Example 3.1.4 (1), p. 82]. Now, assume gl. $\operatorname{dim} R=$ 2 .

(i) Since gl. $\operatorname{dim} R_{M} \leq 2$ for each maximal ideal $M$ of $R, R_{M}$ is a coherent $G C D$ domain [14, Theorem 2.2]. By Proposition 2.2, $R$ is a coherent ring. $\forall a, b \in R$, by Proposition 2.1, $p d_{R_{M}}(a, b)_{M} \leq 1$. Hence $p d_{R}(a, b)=$ flat- $\operatorname{dim}_{R}(a, b)=\sup _{M}\left\{\right.$ flat- $\left.\operatorname{dim}_{R_{M}}(a, b)_{M}\right\}=\sup _{M}\left\{p d_{R_{M}}(a, b)_{M}\right\} \leq 1$, and then $R$ is a $G C D$ domain.

(ii) Since gl. $\operatorname{dim} R_{M} \leq 2$ for each maximal ideal $M$ of $R$, each ideal of $R_{M}$ is countably generated [14, Theorem 4.8], and then so is each ideal of $R$, by Proposition 2.2.

(iii) First, we have that f.p. $\operatorname{dim} R \neq 2$, or else there exists some ideal $I$ of $R$ such that f.p. $\operatorname{dim}_{R}(I)=1$ [8, Corollary 2.9]. Hence for each maximal ideal $M$ of $R$. f.p. $\operatorname{dim}_{R_{M}}\left(I_{M}\right) \leq 1$, but f.p. $\operatorname{dim} R_{M} \neq 2$, so f.p. $\operatorname{dim}_{R_{M}}\left(I_{M}\right)=0$ which means that $I_{M}$ is finitely presented. By Proposition 2.2 (i), f.p. $\operatorname{dim}_{R}(I)=0$; thus we get a contradiction.

From [8, Theorem 3.4] we have that

gl. $\operatorname{dim} R=\sup \{$ Tor- $\operatorname{dim} R$, f.p. $\operatorname{dim} R-1\}$ 
for $R$ is coherent. If gl. $\operatorname{dim} R=2$, then either Tor- $\operatorname{dim} R=1$ and f.p. $\operatorname{dim} R$ $=3$, Tor- $\operatorname{dim} R=2$ and f.p. $\operatorname{dim} R=2$, or Tor- $\operatorname{dim} R=2$ and f.p. $\operatorname{dim} R=$ 3 , and the theorem is proved.

Finally, we give

Theorem 2.2. Let $R$ be an indecomposable semilocal ring of global dimension 2. Then

(i) $R$ is a $(1,2,3)$-ring if and only if each maximal ideal of $R$ is either principal, or nonfinitely generated if and only if $R$ is a Bezout domain.

(ii) $R$ is $a(2,2,0)$-ring if and only if $R$ satisfies the ascending chain condition on principal ideals if and only if $R$ is noetherian.

(iii) In addition, if $J$ is finitely generated, then $R$ is a $(2,2,3)$-ring if and only if there exists a maximal ideal which is not principal, and $R$ is not completely integrally closed.

Remarks. A domain $R$ with quotient field $K$ is completely integrally closed if, for $a$ and $u$ in $K$ with $a \neq 0, a u^{n} \in R$ for all $n$ implies $u \in R$.

Proof. (i) By Theorem 2.1, $R$ is a coherent ring. Assume $R$ is a $(1,2,3)$ ring; then $R$ is a Bezout domain [2, Theorem 4], and then each maximal ideal is either principal or nonfinitely generated.

For the converse, if each maximal ideal $M$ of $R$ is either principal, or nonfinitely generated, then so is $M_{M}$, by Proposition 2.2. Hence, by [14, Theorem 2.2], $R_{M}$ is a valuation domain, which implies that $\operatorname{Tor}-\operatorname{dim} R=$ $\sup _{M}\left\{\right.$ Tor- $\left.\operatorname{dim} R_{M}\right\} \leq 1$. But gl. $\operatorname{dim} R=2$, by Theorem $2.1 R$ is a $(1,2,3)$ ring.

(ii) It is clear that $R$ is a $(2,2,0)$-ring if and only if $R$ is a noetherian ring. Now, assume $R$ satisfies the ascending chain condition on principal ideals. One wishes to prove that $R$ is a noetherian ring. By [14, Theorem 8 ] it is enough to show that each prime ideal of $R$ is finitely generated. Let $P$ be a prime ideal of $R$. By [2, Corollary 4], $R$ is $U F D$. Hence, when $P$ is maximal, there exists an element $d$ in $P$ such that $P=(d)$. When $P$ is not maximal, by Theorem 2.1, $R$ is a coherent $G C D$ domain with global dimension 2, again by the proof of $[14,(7)$, p. 21$], P$ is a directed union of principal ideals. Assume $S=\{(a) \mid a \in P\}$, and apply Zorn's Lemma to $S$. There exists a maximal element (b) in $S$. For any element $a$ in $P$, there exists $c$ in $P$ such that $b \in(c)$ and $a \in(c)$. Hence $(b)=(c)$, which shows that $P=(b)$.

(iii) For the sufficiency, it follows from (i) that $R$ is not a $(1,2,3)$-ring. Observe that $R$ is a GCD domain, and then $R$ is integrally closed [4, Theorem 50]. If $R$ is a $(2,2,0)$-ring, then $R$ is an integrally closed noetherian domain, and then $R$ is completely integrally closed by [4, Example 12, p. 53], a contradiction. Consequently, $R$ is a $(2,2,3)$-ring by Theorem 2.1 .

For necessity, assume $R$ is a $(2,2,3)$-ring. By (i) there exists a maximal ideal of $R$ which is not principal, so we need only to show that $R$ is not completely integrally closed. If this were not so, then $R_{M}$ is completely integrally 
closed for each maximal ideal $M$ of $R$. Observe that $\operatorname{gl} \operatorname{dim} R_{M} \leq 2$. When gl. $\operatorname{dim} R_{M} \leq 1, R_{M}$ is noetherian. When $\operatorname{gl} \operatorname{dim} R_{M}=2$, if $M$ is a principal ideal, by (i) $R_{M}$ is a $(1,2,3)$-ring which shows $R_{M}$ is a completely integrally closed valuation domain, and then Krull- $\operatorname{dim} R_{M} \leq 1$ [4, Example 12, p. 53]; hence, $R_{M}$ is noetherian; if $M$ is not principal, then $R_{M}$ is also noetherian [13, theorem]. Consequently, for any maximal ideal $M$ of $R, R_{M}$ is noetherian. By Proposition 2.2, $R$ is noetherian, and we get a contradication; therefore, $R$ is not completely integrally closed.

Note. It is suggested by the referee that if a semilocal ring $R$ has the property that gl. $\operatorname{dim} R_{P} \leq r$, for each maximal ideal $P$, then gl. $\operatorname{dim} R \leq r$. The authors express their thanks.

\section{REFERENCES}

1. M. F. Atiyah and I. G. MacDonald, Introduction to commutative algebra, Addison-Wesley Reading, MA, 1969.

2. C. Fuchang and Z. Yicai, On the structure of coherent FP-rings, Ann. Chinese Math. (to appear).

3. X. Jinzhong, The modules over a semilocal ring and their homological dimension, Ann. Chinese Math. 6 (1986), 685-691.

4. I. Kaplansky, Commutative rings, Univ. of Chicago Press, Chicago, 1974.

5. __ Fields and rings, Univ. of Chicago Press, Chicago, 1969.

6. H. Matsumura, Commutative algebra (2nd ed.), Benjamin/Cummings, 1980.

7. H. K. Ng, Coherent rings of dimension two, Proc. Amer. Math. Soc. 91 (1984), 518-522.

8. Finitely presented dimension of commutative rings and modules, Pacific J. Math. 113 (1984), 417-431.

9. D. C. Northcott, A first course of homological algebra, Cambridge University Press, Cambridge, 1973.

10. $\ldots$, An introduction to homological algebra, Cambridge University Press, Cambridge, 1960.

11. G. Raynaud, Critères de platitude et de projectivité, Invent. Math. 13 (1971).

12. J. J. Rotman, An introduction to homological algebra, Academic Press, New York, 1979.

13. W. V. Vasconcelos, The local rings of global dimension two, Proc. Amer. Math. Soc. 35 (1972), 381-385.

14. __ The rings of dimension two, Marcel Dekker, 1976.

Department of Mathematics, Guangxi Teachers University, Gui Lin, China 